\title{
Healthy Skepticism and Due Process
}

\author{
Kevin T. Powell, MD, PhD, FAAP' ${ }^{*}$, Lisa B Zaoutis, MD, FAAP²
}

${ }^{1}$ Self employed, locum tenens; ${ }^{2}$ Department of Pediatrics, The Children's Hospital of Philadelphia, Perelman School of Medicine of the University of Pennsylvania, Philadelphia, Pennsylvania.

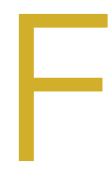

or more than 75 years, pediatrics has sought sound guidelines for prescribing maintenance intravenous fluid (mIVF) for children. In 1957, Holliday and Segar $(H \& S)^{1}$ introduced a breakthrough method for estimating mIVF needs. Their guidelines for calculating free-water and electrolyte needs for mIVF gained wide-spread acceptance and became the standard of care for decades.

Over the last two decades, awareness has grown around the occurrence of rare, life-threatening hyponatremic conditions, especially hyponatremic encephalopathy, in hospitalized children. Concomitantly, an increasing awareness shows that serum levels of antidiuretic hormone (ADH) are often elevated in sick children and triggered by nonosmotic conditions (pain, vomiting, perioperative state, meningitis, and pulmonary disease). This situation led to heightened concern of clinicians and investigators who assumed that hospitalized patients would exhibit reduced tolerance for hypotonic mIVF the mainstay of the H\&S method. The possibility that the H\&S method could be a significant contributing factor to the development of hyponatremic encephalopathy in hospitalized children became a research topic. This research speculated that even mildly reduced serum sodium levels might be a marker for the much rarer condition of hyponatremic encephalopathy. A number of hospitalists also switched from quarter-normal to half-normal saline in mIVF.

The substitution of hypotonic fluids with isotonic fluids (eg, $0.9 \%$ normal saline or lactated Ringer's) is the current front-runner alternative to increase sodium delivery. The hypothesis is that the delivery of additional sodium, while maintaining the same H\&S method volume/rate of fluid delivery, will protect against life-threatening hyponatremic events.

The challenge we face is whether we are moving from mIVF therapy, which features a long track record of success and an excellent safety profile, to a safer or more effective therapeutic approach. We should consider the burden of proof which should be satisfied to support creating new guidelines which center on changing from hypotonic mIVF to isotonic mIVF.

Is there sufficient scientific proof that isotonic mIVF is safer and/or more effective than hypotonic mIVF in preventing life-threatening hyponatremic events?

Is there compelling biologic plausibility for this change for

*Address for correspondence: Kevin Powell, MD, PhD FAAP, 4466 W Pine Blvd Apt 16b, Saint Louis, MO 63108-2339; E-mail: kpowell@alum.mit.edu

Received: June 13, 2018; Accepted: June 21, 2018

๑ 2018 Society of Hospital Medicine DOI 10.12788/jhm.3072 patients with risk factors that are associated with elevated serum ADH levels?

What is the magnitude of the benefit?

What is the magnitude of unintended harms?

We offer our perspective on each of these questions.

The primary difficulty with addressing the adverse events of catastrophic hyponatremia (encephalopathy, seizures, cerebral edema, and death) is their rarity. The events stand out when they occur, prompting mortality and morbidity (M\&M) conferences to blunder into action. But that action is not evidence-based, even if a rationale mentions a meta-analysis, because the rationales lack estimates of the number needed to treat (NNT) to prevent one catastrophic event. Estimates of the NNT to prevent mild hypernatremia are not useful. Furthermore, estimates of the number needed to harm $(\mathrm{NNH})$ via unintended consequences of infusing extra sodium chloride are unavailable. True evidence-based medicine (EBM) is rigorous in requiring NNT and NNH. Anything less is considered M\&M-based medicine masquerading as EBM.

No technical jargon distinguishes the profound and catastrophic events from the common, mild hyponatremia frequently observed in ill toddlers upon admission. As an analo$g y$, in dealing with fever, astute pediatricians recognize that a moderate fever of $103.4^{\circ} \mathrm{F}$ is not halfway to a heatstroke of 108 ${ }^{\circ} \mathrm{F}$. Fever is not a near miss for heatstroke. Physicians do not recommend acetaminophen to prevent heatstroke, although many parents act that way.

No published randomized controlled trials (RCTs) showed the incidence of these catastrophic hyponatremic events. In the meta-analysis of 10 disparate and uncoordinated trials in $2014,{ }^{2}$ no serious adverse events were noted among the 1,000 patients involved. Since then, newer RCTs have added another 1,000 patients to the meta-analysis pool, but still no serious adverse event has been observed.

The H\&S method features 60 years of proven safety and remains the appropriate estimate when composing long-term parenteral nutrition. No recommendation is perfect for all situations. Many hospitalized children will exhibit an increased level of ADH. A very small fraction of those children will present a sufficiently elevated $A D H$ level long enough to risk creating profound hyponatremia. An approximation is in the order of magnitude of 1 per 100,000 pediatric medical admissions and 1 per 10,000 postoperative patients. With 3 million pediatric admissions yearly in the United States, such numbers mean that large children's hospitals might see one or two catastrophic adverse events each decade due to mIVF in previously healthy children. The risk in chronically ill children and in the ICU will 
be higher. The potential for causing unintended greater harm amongst the other millions of patients is high, requiring application of the precautionary principle.

Thus, EBM and RCTs are poor methodologies for quality improvement of this issue. Assigning surrogate measures, such as moderate hyponatremia or even mild hyponatremia, to increase sensitivity and incidence for research purposes lacks a validated scientific link to the much rarer profound hyponatremic events. The resulting nonvalid extrapolation is precisely what true EBM seeks to avoid. A serum sodium of $132 \mathrm{mEq} / \mathrm{L}$ is not a near miss. The NNT to prevent the catastrophic events is unknown. Indeed, no paper advocating adoption of isotonic mIVF has even ventured an approximation.

The RCTs are also, therefore, underpowered to identify harms from using normal saline as a maintenance fluid. A few studies mention hypernatremia, but serum sodium is not a statistical variable. Renal physiology predicts that kidneys can easily handle excess infused sodium and can protect against hypernatremia. However, the extra chloride load risks creating hyperchloremic acidosis, particularly when a patient with respiratory insufficiency cannot compensate by lowering $\mathrm{pCO}_{2}$ through increased minute ventilation. Edema is another risk. Both respiratory insufficiency and edema already occur more frequently (by orders of magnitude) in hospitalized patients on any mIVF than the profound hyponatremia events in hospitalized patients on hypotonic mIVF. For instance, about $1 \%$ of hospitalized infants with bronchiolitis are ventilated for respiratory failure. If hyperchloremic acidosis unintentionally caused by isotonic mIVF slightly increases the frequency of intubation, then such result far outweighs any benefit from reducing catastrophic hyponatremic events. Difficulty will also arise in detecting this unintended increase in the rate of intubation compared with the current background frequency. Detecting these unintended harms becomes impossible if the RCT is underpowered by 100 -fold due to utilizing a surrogate measure, such as serum sodium $<135 \mathrm{mEq} / \mathrm{L}$, as the dependent variable instead of measuring serious hyponatremic adverse events.

All claims that "no evidence of harm" was found from using normal saline as mIVF are type II statistical errors. There is little chance of detecting any harm with a grossly underpowered study or a meta-analysis of 10 such studies. Simply put, EBM is impossible to use for events that occur less than 1 per 10,000 patients using RCTs with 1,000 patients. No usable safety data are available for normal saline as mIVF in any published RCT. As the RCTs are underpowered, one should rely on science to guide therapy, rather than on invalid statistics.

Using the precautionary principle, hypothetically, adding extra sodium chloride to maintenance fluids should be considered in the same manner as adding any other drug. Based on the current evidence, would the Food and Drug Administration approve the drug intravenous sodium chloride for the prevention of hyponatremia induced by maintenance fluids? An increasing evidence of a minimal beneficial effect is observed, but no evidence of safety nor physiology is available. A new drug application for using normal saline as a default maintenance fluid would be soundly rejected by an FDA panel, just as it has been reject- ed by the majority of pediatric hospitalists throughout the past 15 years since the idea was proposed in 2003.

With the lack of compelling statistical evidence to guide practice, clinicians often rely on biologic plausibility. Relatively recent studies have revealed that many sick children develop elevated blood levels of ADH due to nonosmotic and nonhemodynamic triggers. Fortunately, we also possess a strong body of knowledge around management of children with syndrome of inappropriate secretion of antidiuretic hormone (SIADH). We understand that elevated levels of ADH in the blood causes an increase in the resorption of free water from the renal collecting tubules. No increase in loss of renal sodium nor chloride is associated with this hormonal influence. The resultant hyponatremia is due to excess free-water retention and not the excess loss of sodium or chloride. To manage this condition, patients are not given a salt shaker and then allowed to drink ad libitum. The standard and well-accepted management of patients with $\mathrm{SIADH}$ is the restriction of free-water intake because this step addresses the dysfunctional renal process. Administering sodium chloride to a child with SIADH might possibly slow down the progression of hyponatremia but would also expand the total fluid volumes of the patient and would indirectly deal with a problem that could be addressed directly.

Understandably, in an intensive care setting, when hemodynamics is dicey, and when fluid-restriction could risk hypovolemia, employing a volume-expanding solution for mIVF therapy might be reasonable. However, in an ICU setting, SIADH is routinely treated with free-water restriction, and careful calculations of an individual patient's fluid and electrolyte losses and needs are made.

In conclusion, we recognize the motivation for questioning the $\mathrm{H} \& \mathrm{~S}$ method for mIVF as our field surveilles more than a half-century of accumulated experience with this method and the advances in our understanding of physiology and pathophysiology. However, we believe that the current body of evidence fails to substantiate the proposed recommendations. ${ }^{3}$ The avoidance of laboratory-detectable decreases in serum sodium levels is an unproven marker for the development of life-threatening hyponatremic events. Concerns for untoward effects (eg, excessive volume expansion and effects of hyperchloremia toward acidosis) and the exploration of alternative approaches (eg, modifications in volumes/rates of fluid delivery) have been inadequately explored. The proposed changes in practice may provide no mitigation in the rare events we hope to avoid, may fail to serve all subpopulations within the proposed scope of patients, and will likely create unintended new problems.

Disclosures: Dr. Powell and Dr. Zaoutis have nothing to disclose.

\section{References}

1. Holliday MA, Segar WE. The maintenance need for water in parenteral fluid therapy. Pediatrics 1957;19(5):823-832.

2. Wang $J, X u E, X i a o ~ Y$. Isotonic versus hypotonic maintenance IV fluids in hospitalized children: a meta-analysis. Pediatrics 2014;133(1):105-113. doi: 10.1542/peds.2013-2041

3. Hall AM, Ayus JC, Moritz ML. The default use of hypotonic maintenance intravenous fluids in pediatrics. J Hosp Med. 2018;13(9)637-640. doi: 10.12788/ jhm.3040. 